\title{
An Improved Cluster Routing Structure of IOT
}

\author{
Zhihui Wang*, Ruokun Wu, Qila Sa, Jinlin Li,Yuejiao Fan, Wenbo Xu and Yiqun Zhao \\ Dept of Electronic Engineering, College of Electronic Information and Engineering, \\ Inner Mongolia University, Hohhot 010021, China \\ *Corresponding Author
}

\begin{abstract}
Nowadays, the Internet of things (IOT) is becoming more and more popular. Due to routing protocol easy to extend, hierarchical routing protocol, for example Low Energy Adaptive Clustering Hierarchy (LEACH), is more widely used. In order to lengthen the life of network as long as possible, an improved rotation mechanism of cluster head based on LEACH is proposed in this paper. The main idea is to minimize the reconstruction of the cluster, and change the cluster head in original cluster. We adopted token here. The simulation experimental results on NS II platform show that the improvement can delay the death time of the first node, which makes the energy consumption of the whole network more average. The proposed improved mechanism is effective.
\end{abstract} token

Keywords-internet of things; leach protocol; cluster head; slot;

\section{INTRODUCTION}

IOT (Internet of Things) is a very hot research field in networking. Because of lower prices and increasingly powerful sensor function, its scope of application has gradually penetrated into all aspects of daily life from the military field, such as logistics, health care, agricultural production, smart house, and so on. Almost IOT can be applied to all of the local people's lives [1].

The concept of IOT first appeared in 1991. Bill Gates in his book $<$ The Road Ahead $>$ also mentioned the IOT, but limited by the technical condition, the IOT did not attract public attention. In 2004, Japan first proposed the u-Japan plan. This plan want to make Japan becoming a society that things and things, human and things can communicate everywhere in the future. South Korea and the European Union also made a similar plan in the next few years. When Obama became president, the United States had even more IOT included in the national strategic plan construction. Since then the IOT entered a period of rapid development [2].

Although IOT is known as the net, but is a great different with our daily Internet. It has its own hierarchy, proprietary technology and features.

Some scholars specifically focus on the research and design of the structure or topology of the Internet of things [3-5].

Since it is a network, of course, is inseparable from the protocol and routing algorithm. Therefore, more and more researchers focus on the research of networking protocol and routing algorithm. There are mainly data centric-based routing [6], routing protocol based on geographic information [7], multi path-based routing protocol [8], hierarchy-based Routing Protocol, and so on [9-11].

Based on hierarchical cluster routing structure, this paper aimed to extend the life cycle of IOT as far as possible, and proposed an improved LEACH (Low Energy Adaptive Clustering Hierarchy) protocol structure.

\section{INTERNET OF THINGS}

\section{A. The Structural Framework of IOT}

The target of IOT is establishing the connection of real word and network data. It mainly gets object data by RFID, GPS, sensor and other equipments. And then the data will be managed by the network. The IOT uses existing network and new techniques to extend the concept of network. The IOT is changing our life. In the future, network will full coverage of our lives.

Usually the structural framework of IOT consists of three layers: sensor layer, network layer and application layer [12]. Sensor layer is mainly used to collect and identify the information, and it can also carry out some simple processing. Its key technique contains sensor technique, self-organizing network, GPS and some other techniques. Network layer is in charge of sending message. It provides access for sensor layer to the public network which includes telecommunication network and World-Wide-Web. Application layer facing all kinds of application, connects the users and complete the users final purpose.

\section{B. Network Protocol Model}

Net topology can be divided into two types: flat routing protocol and hierarchical routing protocol.

Flooding protocol is a representative of flat routing protocol [13]. It take the broadcast way to transmit information. Its advantage is as long as there is path, the information can be delivered. Its disadvantage is also obvious: too much redundant information circulate in the network. The bigger the network size is, the shorter the network life is.

LEACH protocol is a representative of hierarchical routing protocol. LEACH protocol use tree structure. The whole network consists of some clusters, each cluster have a cluster head node. The head node is responsible for communicating the normal node and the sink node, also sometimes processing the information [14]. 
Compared the two structures, hierarchical routing protocol is easy to expand, and have longer life.

\section{METHODOLOGY}

\section{A. LEACH Protocol}

LEACH protocol has a circle implementation process, called Rounds. Each Rounds have two phases: Set-up phase and Steady-State phase.

During Set-up phase, cluster head is elected, then it makes some nodes into its cluster. We presume every node has same energy. The number of the cluster head is $P_{\text {opt }} N . N$ is the number of total node. $P_{\text {opt }}$ is the proportion of cluster head node. $T\left(S_{\boldsymbol{i}}\right)$ decide which node can be cluster head.

$$
T\left(S_{\boldsymbol{i}}\right)= \begin{cases}\frac{\boldsymbol{P}_{\text {opt }}}{1-\boldsymbol{P}_{\text {opt }}\left(r \bmod \left(\frac{\mathbf{1}}{\boldsymbol{P}_{\text {opt }}}\right)\right)}, & \text { if } \quad S_{i} \in G \\ \mathbf{0} & \text {,otherwise }\end{cases}
$$

where $R$ the current Round, $G$ is the set of node which was not be cluster head at that time. Every node has a random number between $[0,1]$. If the random number is greater than $T\left(S_{i}\right)$, the node will be cluster head. In this way, every node has chance to be cluster head, so energy consumption will be more uniform.

When the cluster head is selected, it will send message to other node telling it is the cluster head. Each node decide to join one of clusters depending on the distance between cluster head and itself. In order to prevent the conflict the cluster head use TDMA scheme to allot time. When all of the node have gotten the scheme, the network comes into Steady-State Phase.

During Steady-State Phase, normal node which is not on working will come into dormant mode. LEACH protocol divides time into some frames, each node can only transfer data in prescribed frame.

\section{B. Proposed Improved Protocol Based on LEACH}

Although LEACH protocol lengthen the life of network, it still has some problems. When a Round is over, cluster will rebuild. But rebuilding cluster will use a lot of energy. So we want the time of each round could be enough long. Lengthen the time of each round is feasible, but it will bring other problems. Since the cluster head is responsible for communicating, it cost more energy than normal node. If only lengthen the time of each round, this will lead to the head node's energy depletion, and the network will reduce the stability. Our plan is to minimize the reconstruction of the cluster, change the cluster head in original cluster. Considering the PEGASIS protocol, we decide to use token. When the first time cluster construct, every cluster will put a schedule decided the sequence that which node at what time to be the cluster head. There is three parameters: $T_{\text {round }}, \tau_{\boldsymbol{a}}, \tau_{\boldsymbol{b}} . T_{\text {round }}$ is the time of cluster rebuilt which decide on the actual situation. $\tau_{\boldsymbol{a}}$ is a network parameter that is greater than frame length divided by the channel rate. $\tau_{\boldsymbol{b}}$ is $\tau_{\boldsymbol{a}}$ multiply by the number of normal node in the cluster. $\tau_{\boldsymbol{b}}$ is also the time of each node being the cluster head

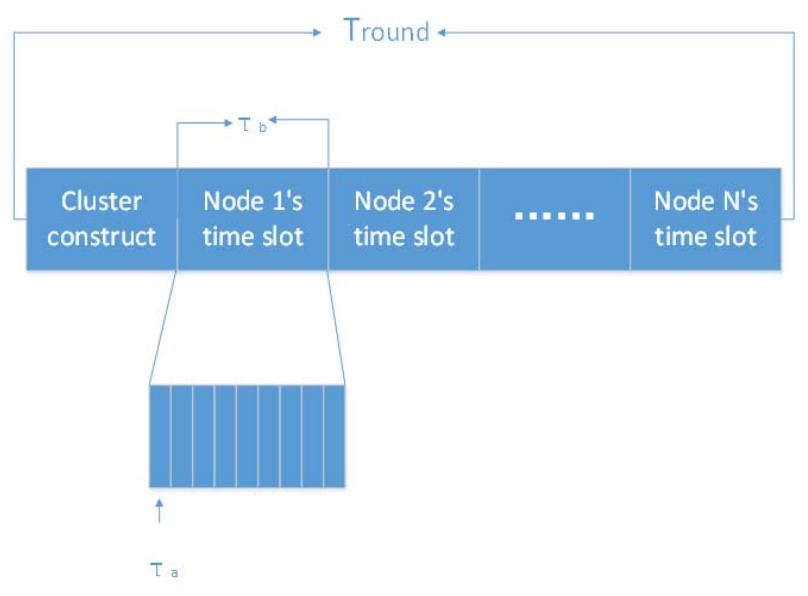

FIGURE I. IMPROVED TIME SCHEDULE

When cluster has constructed, cluster head counts the number $\mathrm{N}$ of normal node in its cluster, and assigns a sequence number for each node like figure 1 . In first time slot, the working mechanism is same like the normal LEACH protocol. In second time slot, the original cluster head become a normal node, the node 2 takes the token and become cluster head and so on. Since $T_{\text {round }}$ and $\tau_{\boldsymbol{a}}$ is a default number, in some clusters few node has no chance to be cluster head, and in some clusters few node should be cluster head more than one time.

When the time of $T_{\text {round }}$ is over, the cluster will rebuild like normal LEACH protocol.

\section{Simulation Result and Performance Analysis}

Through the NS2 network simulation platform, we have carried out experiments on the proposed improvement scheme.

NS2, which full name of is Network Simulator version 2, is a technology for network simulation platform. NS2 is a kind of oriented object of network simulator, it is actually a discretetime simulator which is internally provided with a clock and all simulation are composed of discrete event driven. It contains simulation event scheduler, network component library. Simulation event scheduler controls the process of simulation, and network component objects simulates communication network devices or nodes [15]. 


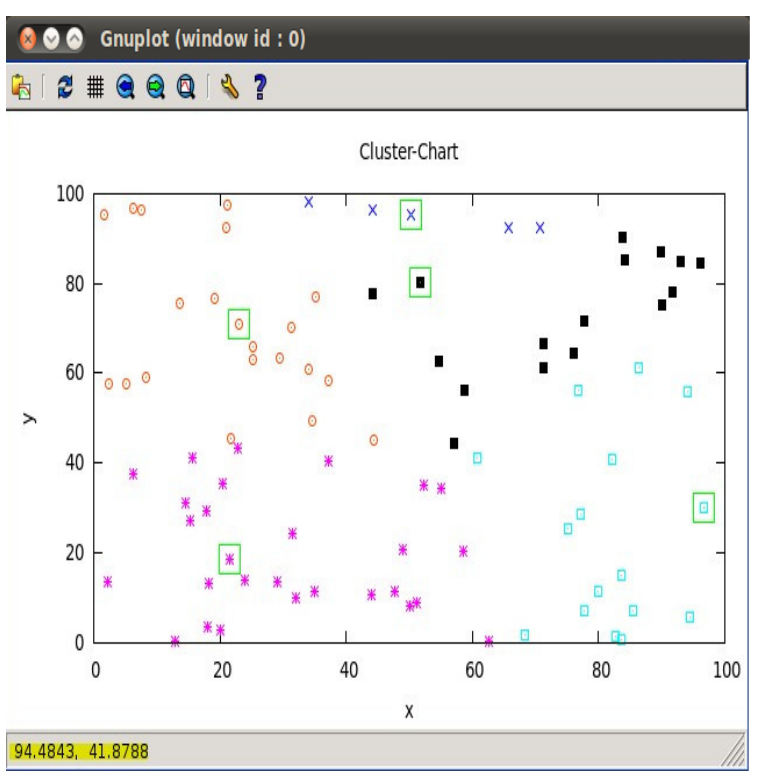

FIGURE II. SCHEMATIC DIAGRAM OF NODES DISTRIBUTION
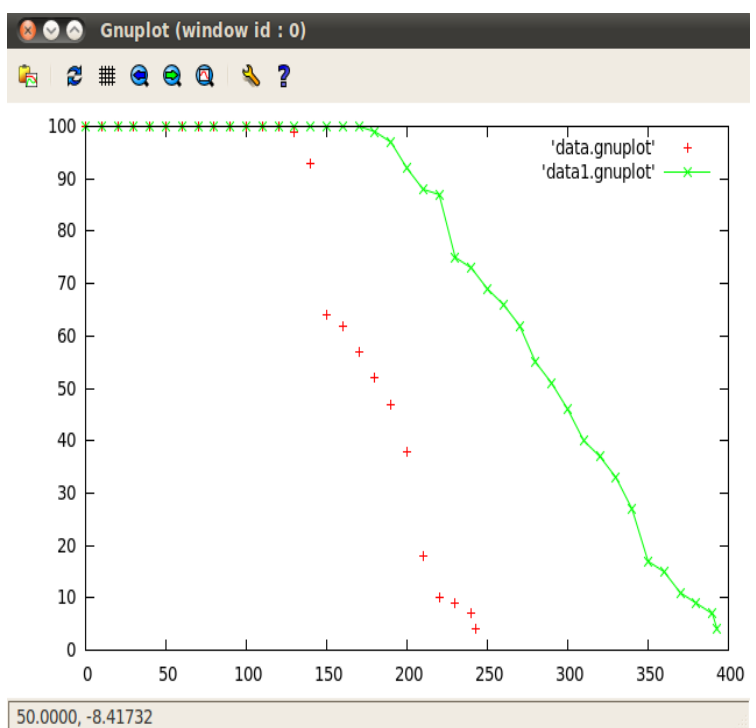

FIGURE III. SCHEMATIC DIAGRAM OF ALIVE NODES

Figure 2 shows the distribution of all nodes. The nodes having the same shape are in the same cluster. The node in a square is the cluster head in this round. We set 100 nodes in a map whose size is $100 \times 100$, and sink node locates at [5,17.5].

Figure 3 shows the node alive in the same situation. The red one named 'data. gnuplot' is corresponding to normal LEACH. The green one named 'data1.gnuplot' denotes our improved LEACH. We can find that the death of node starts at 130 in the normal LEACH, and all nodes have been dead at 250 nearly. But in advanced LEACH, the dead node appears at 170, and at 400 almost all nodes die. After verification the life of network have been extended exactly. In this paper, the cluster head election does not take into account the residual energy of the node, which can be further discussed in the following studies.

\section{CONCLUSIONS}

Clustering algorithm is propitious to expend the scale of network. Without use of location aided case, hierarchy structure will save more energy than the plane structure. Through lengthening the cycle time of LEACH protocol, we reduce the energy consumption of the whole cluster, and the network lifetime is prolonged. Simulation experimental results show that the improvement can delay the death time of the first node, which makes the energy consumption of the whole network more average.

\section{ACKNOWLEDGMENT}

The research in this paper was sponsored by the National Natural Science Foundation of China (Grant No.61261003), and the Natural Science Foundation of Inner Mongolia Autonomous Region of China (Grant No.2015MS0610).

\section{REFERENCES}

[1] QIAN Zhi-hong, WANG Yi-jun. "IoT Technology and Application", Chinese Journal of Electronics, vol. 40, pp. 1023-1029, 2012.

[2] ITU. The Internet of Things[J]. Whole Earth, vol.297, pp.949-955, 2008.

[3] Song H, Lee S H, Lee S, et al. "6Lo WPAN-based tactical wireless sensor network architecture for remote large-scale random deployment scenarios", IEEE Military Communications Conference, pp. 1-7, 2009.

[4] Hong S, Kim D, Ha M, et al. "SNAIL: an IP-based wireless sensor network approach to the internet of things", Wireless Communications IEEE, vol. 17, pp. 34-42, 2010.

[5] Zhou L, Chao H C. "Multimedia traffic security architecture for the internet of things", Network IEEE,vol. 25, pp. 35-40, 2011.

[6] Ya-lin Nie, San-yang Liu, Zhi-bin Chen, Xiao-gang Qi. "Adaptive routing algorithm based on data aggregation in event-driven wireless sensor networks", Control and Decision, vol. 29, pp. 1477-1482, 2014.

[7] Dejing Zhang. "Research on virtual coordinates-based routing protocol of wireless sensor networks", ShanDong University, 2015.

[8] Chunli Lin, Jie Cui. "Adaptive Multipath Secure Routing Protocol in WSN", Computer Engineering, vol. 42, pp. 144-150, 2016,.

[9] Aristides Mpitziopoulos, and Basilis Mamalis, “A Rendezvous-Based Approach Enabling Energy-Efficient Sensory Data Collection with Mobile Sinks", IEEE Transactions on parallel and distribute systems, vol. 23, NO. 5, 2012.

[10] Haosong Gou and Younghwan Yoo. "A Partition-Based LEACH Algorithm for Wireless Sensor Networks”, IEEE Ninth International Conference on Computer and Information Technology, pp. 11-14, 2009.

[11] Qiong Yang, Song Xing, Weiwei Xia, Lianfeng Shen. "MAC upper band delay based adaptive clustering algorithm for VANET", Journal of Southeast University (Natural Science Edition), vol. 46, pp. 1-6, 2016.

[12] Atzori L, Iera A, Morabito G. "The internet of things: A survey", Computer Networks, vol. 54, pp. 2787-2805, 2010.

[13] Niu J, Cheng L, Gu Y, et al. "Minimum-delay and energy-efficient flooding tree in asynchronous low-duty-cycle wireless sensor networks", Wireless Communications and Networking Conference, IEEE,vol. 2013, pp. 1261-1266, 2013.

[14] Gopi Saminathan Arumugam, Thirumurugan Ponnuchamy. "EELEACH: development of energy-efficient LEACH Protocol for data gathering in WSN", EURASIP Journal on Wireless Communications and Networking, vol. 1, pp.1-9, 2015.

[15] Qiang Wang, Jun Jiao, Wen Kong, Ya Gao, Chen-chen Yuan. "Simulation of wireless sensor networks for fixed and mobile nodes based on NS2", Journal of Hefei University(Natural Sciences Edition), vol. 25, pp. 24-28, 2015. 